ARTICLE

DOI: $10.1038 / \mathrm{s} 41467-018-04523-y$

\title{
Zinc ion flux during mammalian sperm capacitation
}

\author{
Karl Kerns ${ }^{1}$, Michal Zigo ${ }^{1,2}$, Erma Z. Drobnis ${ }^{3}$, Miriam Sutovsky ${ }^{1} \&$ Peter Sutovsky ${ }^{1,3}$
}

Sperm capacitation, the ultimate maturation event preparing mammalian spermatozoa for fertilization, was first described in 1951, yet its regulatory mechanisms remain poorly understood. The capacitation process encompasses an influx of bicarbonate and calcium ions, removal of decapacitating factors, changes of $\mathrm{pH}$ and sperm proteasomal activities, and the increased protein tyrosine phosphorylation. Here, we document a novel biological phenomenon of a unique zinc $\left(\mathrm{Zn}^{2+}\right)$ ion redistribution associated with mammalian sperm in vitro capacitation (IVC). Using image-based flow cytometry (IBFC), we identified four distinct types of sperm zinc ion distribution patterns (further zinc signature) and their changes during IVC. The zinc signature was altered after sperm capacitation, reduced by proteasomal inhibitors, removed by zinc chelators, and maintained with addition of external $\mathrm{ZnCl}_{2}$. These findings represent a fundamental shift in the understanding of mammalian fertilization, paving the way for improved semen analysis, in vitro fertilization (IVF), and artificial insemination (AI).

\footnotetext{
${ }^{1}$ Division of Animal Sciences, University of Missouri, Columbia, MO 65211-5300, USA. ${ }^{2}$ Laboratory of Reproductive Biology, Institute of Biotechnology, Czech Academy of Sciences, v.v.i., 25242 Vestec, Czech Republic. ${ }^{3}$ Department of Obstetrics, Gynecology and Women's Health, University of Missouri, Columbia, MO 65211-5300, USA. Correspondence and requests for materials should be addressed to P.S. (email: SutovskyP@missouri.edu)
} 
A zinc spark (an exocytotic event releasing billions of zinc ions) issued from the oocyte induced by the spermatozoa at fertilization $^{1}$ is implicated as a novel biomarker of mammalian embryo quality and developmental potential ${ }^{2}$. To date, zinc ion $\left(\mathrm{Zn}^{2+}\right)$ fluxes have not been well characterized in mammalian spermatozoa, though sperm-flagellar voltage-gated proton channel HVCN1, negatively regulated by $\mathrm{Zn}^{2+}$, has been implicated as the main proton extrusion mechanism during mammalian sperm capacitation $^{3}$. This channel regulates intracellular $\mathrm{pH}$ and consequently is thought to be responsible for $\mathrm{Ca}^{2+}$ entry via opening of the CatSper channel, all of these events coinciding with PKA activation and the hallmark increase of protein tyrosine phosphorylation during boar sperm capacitation ${ }^{4,5}$. Further, the $26 \mathrm{~S}$ proteasome, a multi-subunit ubiquitin-dependent protease, regulates fertilization at multiple steps from spermiogenesis to sperm penetration of the oocyte zona pellucida $(\mathrm{ZP})^{6}$ including certain aspects of sperm capacitation ${ }^{7}$. In particular, the A-kinase anchoring protein AKAP3 is degraded by the ubiquitin-proteasome system (UPS) during bull sperm capacitation ${ }^{8}$, and the E1-type ubiquitin-activating enzyme (UBA1) inhibitor PYR-41 alters acrosomal remodeling. Additionally, proteasomal inhibitors hinder the capacitation-associated shedding of acrosin-inhibitor serine peptidase inhibitor kazal type 2 (SPINK2) and spermadhesin $\mathrm{AQN1}^{9}$ in the boar (see review ${ }^{7}$ ).

Here, we use image-based flow cytometry (IBFC) to document four distinct types of sperm zinc signature and their changes during in vitro capacitation (IVC) of domestic boar, bull, and human spermatozoa, altered after sperm capacitation, further reduced under proteasome inhibiting IVC conditions, removed by zinc chelators, and maintained with addition of external $\mathrm{ZnCl}_{2}$. The zinc shield established by the oocyte following fertilization could derail such sperm zinc signaling as an added barrier to pathological polyspermic fertilization. This all together supports a new role of zinc ions during capacitation and fertilization. Such findings represent a fundamental shift in the understanding of mammalian fertilization, paving the way for a more accurate semen analysis to ameliorate the methodology of in vitro fertilization (IVF) and artificial insemination (AI).

\section{Results}

Mammalian spermatozoa possess four distinct zinc signatures. We used state-of-the-art IBFC and epifluorescence microscopy to trace the sperm zinc signature using Zn-probe FluoZin ${ }^{\mathrm{Tm}}-3$ AM (FZ3), DNA stain Hoechst 33342, acrosomal remodeling detecting lectin PNA (Arachis hypogea/peanut agglutinin) conjugated to Alexa Fluor $^{\text {TM }} 647$ (PNA-AF647), and live/dead cell, plasma membrane integrity reflecting DNA stain propidium iodide (PI), which is taken up exclusively by cells with a compromised/ remodeled plasma membrane. The IBFC, which combines the fluorometric capabilities of conventional flow cytometry with high speed-multi-channel image acquisition, proved to be advantageous due to the high presence of $\mathrm{Zn}^{2+}$ in sperm cytoplasmic droplets and seminal debris, which otherwise would distort traditional flow cytometry results. We developed a unique gating and masking strategy to ensure unbiased data analysis (Supplementary Fig. 1). Analyses were performed using the initial, pre-sperm-rich fraction of ejaculates, which had highest sperm viability/plasma membrane integrity, repeatability, and sensitivity to proteasomal inhibition compared to secondary, sperm-rich fraction that appeared more prone to spontaneous capacitation and loss of plasma membrane integrity.

We identified four distinct types of sperm zinc signatures conserved across boar (Fig. 1a-d), bull (Fig. 1e-h), and human spermatozoa (Fig. 1i-1): high $\mathrm{Zn}^{2+}$ presence in the sperm head and whole-sperm tail (signature 1; Fig. 1a, e, i), medium-level (based on relative intensity of fluorescence in FlowSight measurements) $\mathrm{Zn}^{2+}$ presence in both the sperm head and

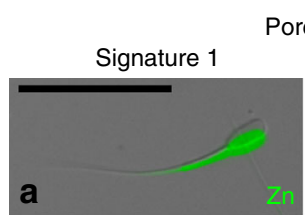

Signature 3

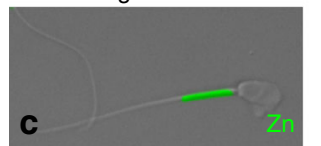

Human

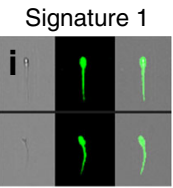

Signature 3

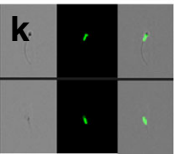

BF Zn Merge

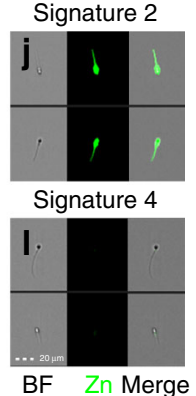

Signature 2

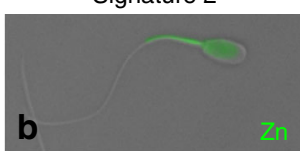

Signature 4

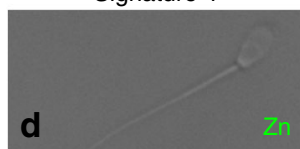

$\mathrm{Zn}$

\section{.}

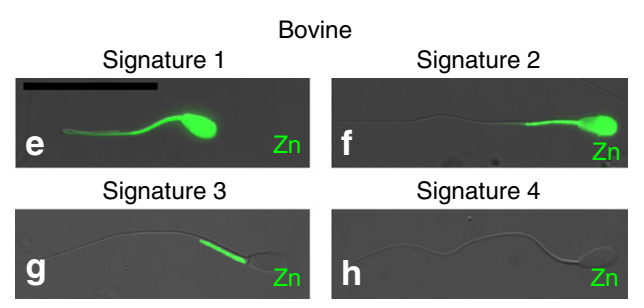

Porcine whole sperm sample population

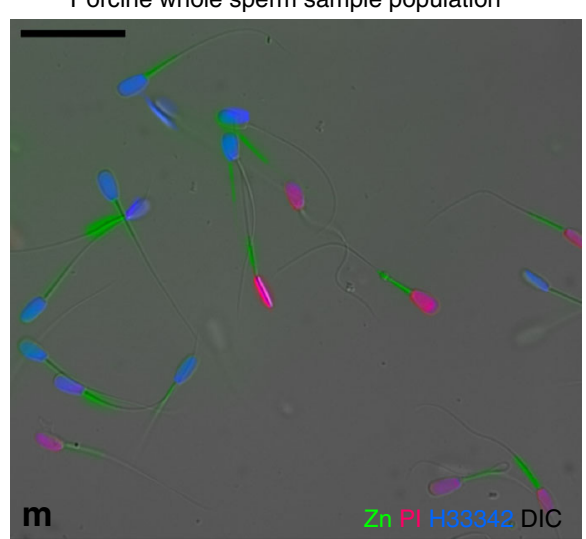

Fig. 1 Mammalian sperm zinc signature. a-d Epifluorescence microscopy of boar sperm zinc signature (green). e-h Epifluorescence microscopy of bull sperm zinc signature. i-I FlowSight image gallery of human sperm zinc signature (scale bar: $20 \mu \mathrm{m}$ ). $\mathbf{m}$ Boar spermatozoa after $72 \mathrm{~h}$ of storage in Beltsville thaw solution (BTS semen extender) show varied zinc signatures. Imprecise fluorescent to bright-field overlay illustrates motile status (all scale bars: $25 \mu \mathrm{m}$, unless noted) 
a

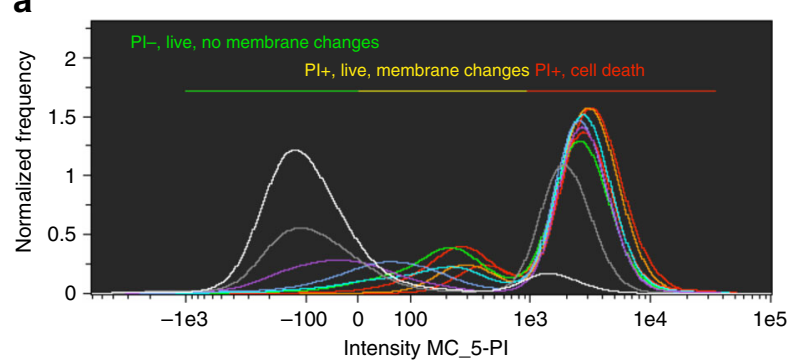

C

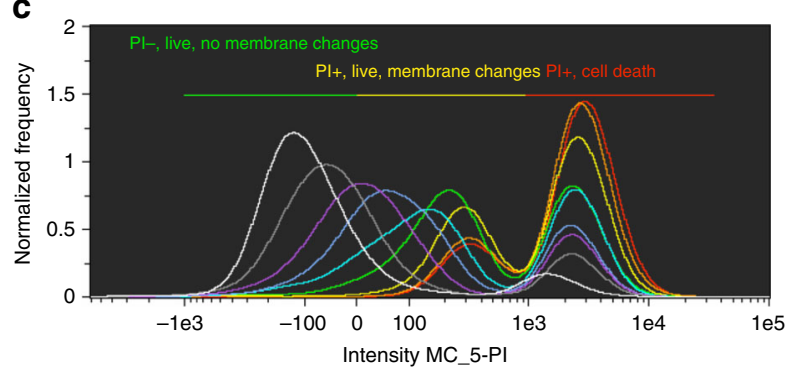

b

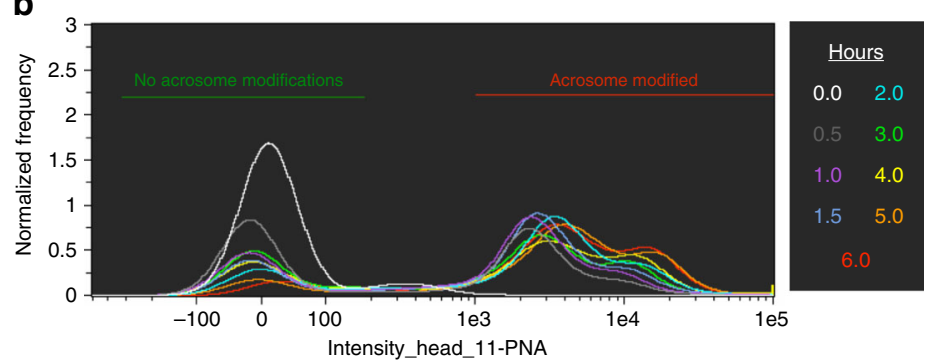

d

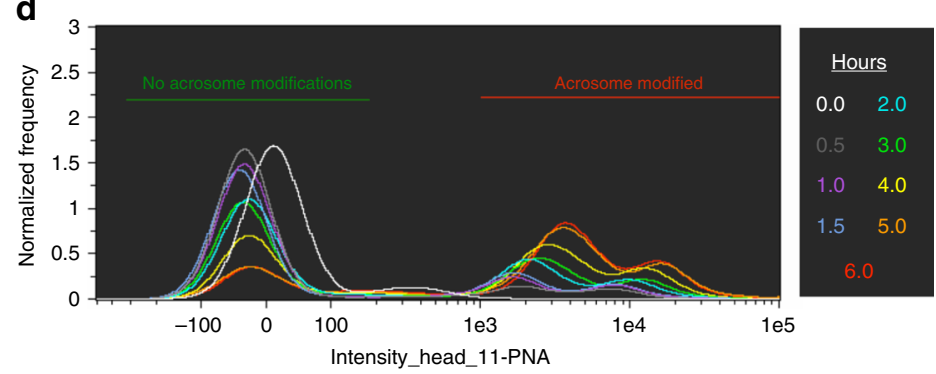

Fig. 2 Zinc signature time course with high vs. low bicarbonate IVC. Time-lapse recordings of zinc signature during IVC in high, $15 \mathrm{mM}$ sodium bicarbonate media: (a) plasma membrane changes as identified by $\mathrm{PI}$ status, distinguishing between IVC-induced $\mathrm{PI}^{+}$subpopulations $\left(\mathrm{PI}+{ }^{+}\right.$live with plasma membrane changes vs. $\mathrm{PI}^{+}$cell death). b Acrosomal modifications. Time course of zinc signature modification in low, 2 mM sodium bicarbonate IVC media: (c) plasma membrane changes, and $\mathbf{d}$ acrosomal modifications (corresponding histogram color code for time points in figure legend)

sperm tail midpiece (signature 2, spermatozoa undergoing capacitation; Fig. $1 \mathrm{~b}, \mathrm{f}, \mathrm{j}), \mathrm{Zn}^{2+}$ presence in the midpiece only (signature 3/capacitated state signature in spermatozoa that underwent capacitation and may be dying; Fig. 1c, g, k), and no $\mathrm{Zn}^{2+}$ presence (signature 4, spermatozoa with compromised/ remodeled plasma membrane; Fig. 1d, h, l). Spermatozoa after 72 $\mathrm{h}$ of storage in Beltsville thaw solution (BTS semen extender) show varied zinc signatures (Fig. $1 \mathrm{~m}$ ).

Zinc signature is indicative of capacitation status in vitro. A drawback to commonly used $15 \mathrm{mM}$ sodium bicarbonate IVC media is rapid sperm death (as compared to in vivo sequential capacitation ${ }^{10}$ ), illustrated in the time course study by a shift to PI

cell death flow cytometry gating (Fig. 2a) and rapid acrosomal modification (Fig. 2b). In the interest of emulating in vivo sperm lifespan and sequential capacitation as a fertility diagnostic method, we used a previously described capacitation medium ${ }^{11}$ with low $(2 \mathrm{mM})$ sodium bicarbonate and increased sodium pyruvate $(5 \mathrm{mM})$ that prolonged sperm viability (Fig. 2c) and elicited similar hyperactivation (Supplementary Movie 1) while achieving hallmark acrosomal modification (Fig. 2d; discussed further in Methods, in vitro capacitation section).

Most spermatozoa in zinc signature 1 and 2 states had no capacitation-like acrosomal remodeling $(93.0 \pm 6.8 \%$ and $95.0 \pm$ $2.6 \%$, data presented as mean \pm s.d.; 10,000 cells analyzed per treatment, $n=3$ biological replicates) compared to zinc signature 3 and $4(11.1 \pm 5.8 \%$ and $7.0 \pm 9.9 \% ; P<0.0001$, as determined by the general linear model (GLM) procedure). Capacitation-like acrosomal remodeling was most prevalent with zinc signatures 3 and $4(81.0 \pm 8.5 \%$ and $62.2 \pm 12.9 \%)$ compared to zinc signatures 1 and $2(4.0 \pm 4.7 \%$ and $3.4 \pm 2.9 \% ; P<0.0001$, as determined by the GLM procedure). Acrosome exocytosis occurred within the subpopulation of spermatozoa with zinc signature $4(30.7 \pm 3.0 \%)$ and was greater than zinc signatures 1,2 , and $3(3.0 \pm 2.6 \%, 1.6 \pm$ $1.2 \%, 7.9 \pm 2.9 \% ; P<0.001$, as determined by the GLM procedure; Fig. 3a; Table 1). As sperm plasma membrane integrity decreased, signaled by increased PI labeling, the zinc patterns progressed to signatures 3 and 4 (Fig. 3b). Hyperactivated spermatozoa, capable of recognizing and binding the oocyte $\mathrm{ZP}$ have zinc signature 2 (Supplementary Movies 2-4), in which the transition from signature 1 to 2 occurs within the first $30-60 \mathrm{~min}$ of IVC (Supplementary Fig. 2m).

$26 S$ proteasome modulates zinc signature capacitation shift. Fresh, ejaculated boar spermatozoa mostly had signature $1,(83.8 \pm$ $3.1 \%$; data presented as mean \pm s.e.m.; 10,000 cells analyzed per treatment, $n=3$ biological replicates; Fig. $4 \mathrm{a}$, e; Table 2). A small portion of spermatozoa incubated in non-IVC media for $4 \mathrm{~h}$ at $37^{\circ}$ $\mathrm{C}$ progressed to signature 2 (Fig. $4 \mathrm{~b}$ ) as compared to spermatozoa in the same media incubated at room temperature to emulate the conditions of AI (Fig. 4a), suggesting that some spermatozoa undergo temperature-induced, early-stage capacitation. When proteasome inhibitor MG-132 was added to IVC conditions to reduce sperm proteasome activity as previously described ${ }^{12,13}$, a significantly higher portion of spermatozoa retained signature 1 when using the pre-sperm-rich fraction (Fig. 4c, d, e) as compared to IVC + vehicle $(P=0.0271$; when signatures 1 and 2 combined $P=0.0008$, as determined by Duncan's multiple range test). After $4 \mathrm{~h}$ of IVC, the zinc signature changed to mostly signature 3 (49.4 $\pm 7.9 \%$ ), with a small portion of spermatozoa having signature 2 $(31.3 \pm 12.3 \%$; Fig. $4 c)$. Remarkably, manipulation of sperm $\mathrm{Zn}^{2+}$ content during IVC reset the zinc signature (Fig. $4 \mathrm{f}, \mathrm{h}, \mathrm{i}$ ). Spermatozoa retained signature 1 with addition of $1 \mathrm{mM} \mathrm{ZnCl}_{2}$ to IVC medium (Fig. 4f). Cell-permeant $\mathrm{Zn}^{2+}$ chelator N,N,N',N'-Tetrakis(2-pyridinylmethyl)-1,2-ethanediamine (TPEN) removed a majority of FZ3 fluorescence to signature 3 and 4 states (Fig. 4g) compared to TPEN vehicle (Supplementary Fig. 3h), but TPEN did not reduce Fluo-4 calcium probe intensity compared to vehicle (Supplementary Fig. 3i). Remaining FZ3 fluorescence is likely due to $\mathrm{Zn}$ presence, but ions tightly bound within the mitochondrial sheath, as zinc has been previously detected there by electron microscopy ${ }^{14}$. With the exception of the midpiece, zinc ions appeared to be associated with the sperm surface, as the stepwise extraction removed $\mathrm{Zn}^{2+}$ tracer fluorescence early in treatment procedure (Supplementary Fig. 3j-1). 


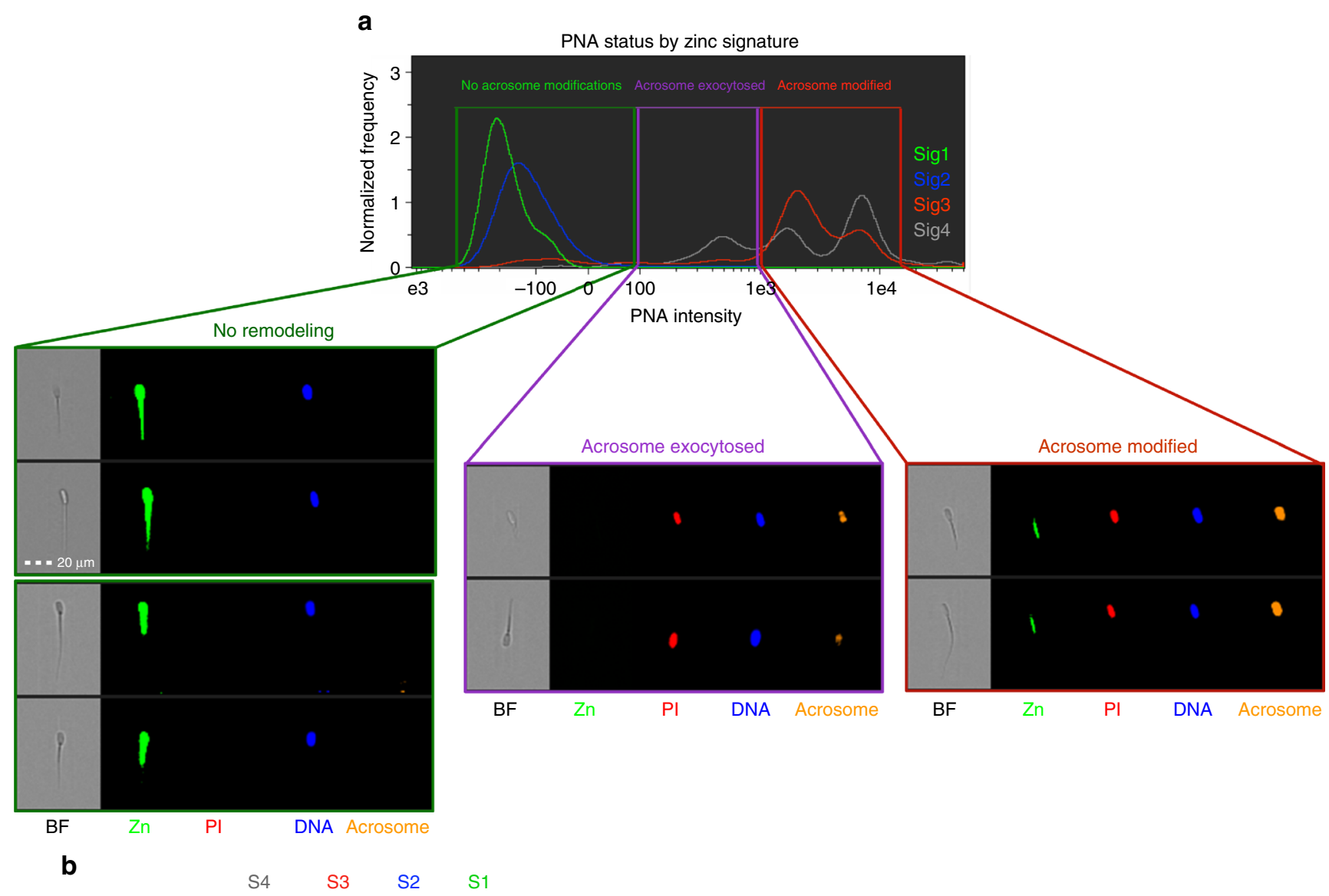

b

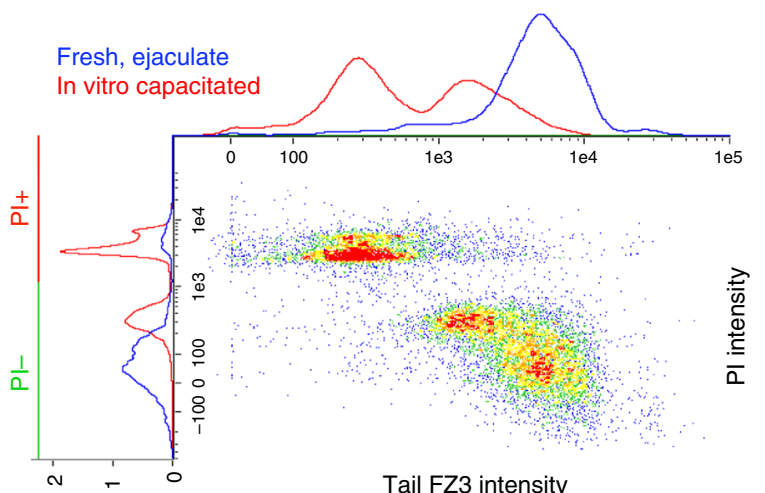

C

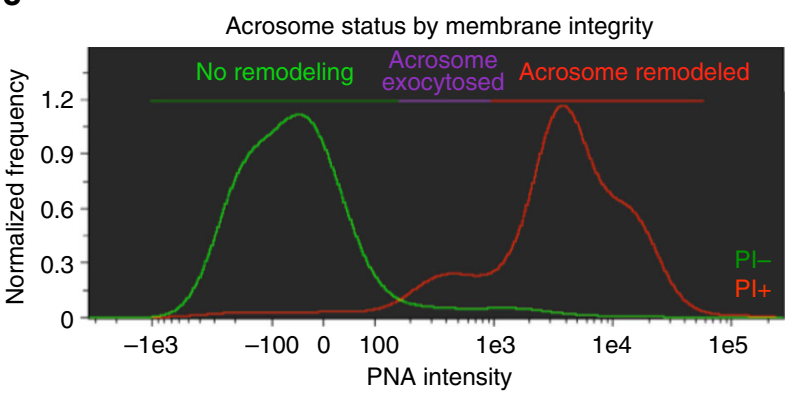

Fig. 3 Acrosomal status and membrane integrity of zinc signature. a Most spermatozoa in zinc signature 1 and 2 states had no capacitation-like acrosomal remodeling compared to zinc signature 3 and $4(P<0.0001$; see Table 1). Capacitation-like acrosomal remodeling was most prevalent with zinc signatures 3 and 4 compared to zinc signatures 1 and 2 ( $P<0.0001 ; 4$ biological replicates; 10,000 spermatozoa analyzed per treatment). Acrosomal exocytosis occurred within the subpopulation of spermatozoa with zinc signature 4 and was greater than zinc signatures 1,2 , and 3 ( $P<0.001$; scale bar: $20 \mu \mathrm{m})$. $\mathbf{b}$ Zinc signature status corresponds with PI plasma membrane integrity in fresh (blue) and IVC spermatozoa (red). c As sperm plasma membrane integrity decreased, acrosomal remodeling and exocytosis occurred. P-values determined by the general linear model procedure in SAS 9.4

Zinc signature associated with varied fertility in AI boars. We examined possible individual variability in sperm zinc signature in AI boars with acceptable but varied fertility. In a small preliminary fertility trial ( $n=4$ boars with known fertility in AI service; fertility records in Supplementary Table 1), zinc signatures differed between high- and low-fertile boars both after IVC (Fig. 5a; original histograms located in Supplementary Fig. 4). Boars with high fertility have double the amount of signature 3 spermatozoa prevail after IVC (as percentage of population) as opposed to minimal signature 3 increase in low-fertility boars (Fig. 5b).

\section{Discussion}

The inhibition of the zinc signature shifts may be related to the presence of Zn-binding UPS enzymes in spermatozoa, such as the RING-finger E3-type ubiquitin ligase UBR7 ${ }^{15}$. The RING (really interesting new gene) finger ubiquitin ligases contain an amino acid motif that binds two zinc cations, allowing interaction with other proteins/enzymes, including establishment of a stable E3substrate interaction required for protein ubiquitination. Other Zn-binding/Zn-containing proteins are likely present in spermatozoa since UBR7 is only detectable in the acrosomal region of fully differentiated spermatozoa and in the centriolar vault of the sperm tail-connecting piece. For example, sperm DNA-binding 


\begin{tabular}{|c|c|c|c|}
\hline & No remodeling & Remodeled & Exocytosed \\
\hline Signature 1 & $93.0 \pm 6.8 \%{ }^{A a}$ & $4.0 \pm 4.7 \% \mathrm{Ba}$ & $3.0 \pm 2.6 \%{ }^{\mathrm{Ba}}$ \\
\hline Signature 2 & $95.0 \pm 2.6 \%{ }^{\mathrm{Aa}}$ & $3.4 \pm 2.9 \% \mathrm{Ba}$ & $1.6 \pm 1.2 \%{ }^{\mathrm{Ca}}$ \\
\hline Signature 3 & $11.1 \pm 5.8 \% \mathrm{Ab}$ & $81.0 \pm 8.5 \%{ }^{\mathrm{Bb}}$ & $7.9 \pm 2.9 \% \mathrm{Aa}$ \\
\hline Signature 4 & $7.0 \pm 9.9 \% \mathrm{Ab}$ & $62.2 \pm 12.9 \%^{\mathrm{Bb}}$ & $30.7 \pm 3.0 \% \mathrm{Cb}$ \\
\hline
\end{tabular}

Data are presented as mean \pm s.d. (three biological replicates). Values with different uppercase superscripts $(A, B, C, D)$ indicate significant difference of the acrosomal status $(P$-value $\leq 0.0001)$ and lowercase superscripts $(a, b, c)$ indicate significant difference of zinc signatures ( $P$-value $\leq 0.0002$ ) as determined by the GLM procedure in SAS 9.4. Both $\mathrm{PI}^{+}$and $\mathrm{PI}^{-}$cells were included in this analysis. A total of 10,000 cells were measured for each replicate

protamine PRM2 contains a zinc-finger domain ${ }^{16}$, which plays a role in stabilization of sperm chromatin and inhibition of transcription. Protamines are believed essential for sperm head condensation and DNA stabilization during spermiogenesis, and alterations in sperm DNA protamination are associated with male infertility $^{17}$. Further, JAMM (JAB1/MPN/Mov34 metalloenzyme) motif of the proteasomal regulatory subunit PSMD14/Rpn11 is a metalloprotease-like $\mathrm{Zn}$ site in the $26 \mathrm{~S}$ proteasome ${ }^{18}$. Matrix metalloproteinases are zinc-dependent enzymes known mainly for their ability to digest extracellar matrix ${ }^{19}$, with MMP-2 and MMP-9 being reported in human seminal plasma ${ }^{20}$. Inhibition of zinc-dependent metalloproteases hindered sperm passage through oocyte vestments during $\operatorname{IVF}^{21}$. Related to aforementioned protamine sperm chromatin stabilization, Zn-ion release from the nuclear zinc bridges described in human spermatozoa could facilitate sperm nucleus decondensation after fertilization, as a prelude to the formation of the zygotic paternal pronucleus $^{22,23}$. Further studies thus should examine the relationship between sperm zinc signature and the aforementioned $\mathrm{Zn}$ containing sperm proteins.

The changes seen in fresh, ejaculated zinc signature and the incubated, non-IVC spermatozoa could be heat-induced during the early stages of capacitation. Temperature influence on capacitation state could be related to the $35-38^{\circ} \mathrm{C}$ thermotropic phase transition identified in the plasma membrane of ram spermato$\mathrm{zoa}^{24}$. These findings are important for livestock semen handling methods prior to AI.

Sperm capacitation, although required for fertility, is a terminal maturation event that leads to rapid cell death unless fertilization occurs $^{10}$. The superimposition of zinc ion labeling and PI labeling in flow cytometric scatter plots allows us to subdivide spermatozoa within the boar ejaculate into four subpopulations (Fig. 3b). Thus, the disproportional representation of signatures 3 and 4, associated with sperm capacitated state and death, may indicate low-fertility ejaculates. Cell membrane changes heralded by PI incorporation in the sperm head at capacitation are concomitant with acrosomal remodeling signaled by lectin PNA binding, even though they occur at the opposite poles of the sperm head (Fig. 3c). As indicated in our time-lapse study, PI intensity changes over the course of capacitation and there are two subgroups of $\mathrm{PI}^{+}$spermatozoa: $\mathrm{PI}^{+}$live with plasma membrane change and $\mathrm{PI}^{+}$cell death (Fig. 2c). Although the signature 2 spermatozoa are the hyperactivated and ZP-interacting ones in Supplementary Movie 2, it is likely that a rapid transition occurs through these last stages of capacitation that cannot be distinguished within limitations of today's technology. After this rapid transition from zinc signature 2 to 3 and associated acrosomal changes, we hypothesize that the final cell death occurs rapidly if fertilization is unsuccessful. This might be the reason for sequential sperm capacitation observed within the oviductal sperm reservoir.
Comparison of zinc signature patterns in boars with varied fertility indicates potential of $\mathrm{Zn}$ probes in the evaluation of livestock sperm quality. While such findings with a small group of boars are preliminary, $\mathrm{Zn}$ fluorometry could be also given consideration in human andrology and infertility diagnostics. For instance, the sperm content of flagellar voltage-gated proton channel HVCN1 varies between human donors ${ }^{3}$. The HVCN1 regulates human CatSper $\mathrm{Ca}^{2+}$ channel localized at the flagellum $^{3}$. With CatSper activation required for hyperactivation and ultimately male fertility ${ }^{25}$, it seems reasonable that zinc signature changes are reflective of this biological event and necessary for preparing the spermatozoa for hyperactivation. Other studies have shown that spermatozoa with higher fertilizing ability have increased changes in capacitation-induced biomarkers ${ }^{26}$. Altogether, this encourages dedicated trials with high statistical power sperm sample sets, aspiring to validate zinc signature as a candidate fertility marker. Such findings not only indicate the existence of sperm subpopulations capable/incapable of fertilizing the oocyte, but even more so that sequential capacitation and resulting waves of sperm release from the sperm reservoir, originally thought to be primarily driven by female reproductive tract-issued signals ${ }^{27-29}$, are rather co-dependent of sperm subpopulation (Fig. 6a, b). Other recent studies hint at the significance of $\mathrm{Zn}^{2+}$ for sperm structure and function/fertility. A decrease in $\mathrm{Zn}$ content of human seminal plasma has been associated with infertility stemming from accidental Chernobyl radiation in Ukraine ${ }^{30}$ and individuals with high levels of asthenoteratozoospermic spermatozoa (low or no motility) have reduced seminal plasma $\mathrm{Zn}$ levels ${ }^{31}$. These findings are consistent with other studies reporting fertile males having increased seminal plasma $\mathrm{Zn}$ levels compared to infertile $\mathrm{men}^{32,33}$. Optimization of semen $\mathrm{Zn}^{2+}$ and/or zinc containing protein(s) levels could thus improve the outcomes of AI in livestock and assisted reproductive therapy in humans.

The signature we describe here is likely representative of $\mathrm{Zn}^{2+}$ being involved in multiple steps of sperm capacitation. At ejaculation, sperm motility is highly dependent upon ionic environment ${ }^{34}$. It is well understood that $\mathrm{Zn}^{2+}$ can modulate cellular signaling, as well as protein kinase and phosphatase activities ${ }^{35}$, and inhibit proteasomal activity ${ }^{36}$. Porcine seminal plasma reportedly contains $1.6-3.6 \mathrm{mM} \mathrm{Zn}^{2+37}$, the highest known levels of $\mathrm{Zn}^{2+}$ found in any bodily fluid, thus likely to be serving some biological function. Further, Zn-binding seminal plasma proteins may protect the sperm plasma membrane against cold shock ${ }^{38}$. As mentioned earlier, $\mathrm{Zn}^{2+}$ negatively regulates $\mathrm{HVCN} 1$ channel, the main proton extrusion mechanism in human spermatozoa ${ }^{3}$. Certain aspects of early capacitation events such as $\mathrm{Ca}^{2+}$ influx require $\mathrm{HVCN} 1$ activation by $\mathrm{Zn}^{2+}$ removal. The $\mathrm{Zn}^{2+}$ content in the human sperm flagella is negatively correlated to sperm motility ${ }^{39}$; however, this may be due to capacitation-induced hyperactivation being inhibited in the presence of high $\mathrm{Zn}^{2+}$. Further, $\mathrm{Zn}^{2+}$-chelator DEDTC has been shown to immobilize spermatozoa, reaffirming the role of $\mathrm{Zn}^{2+}$ in sperm motility ${ }^{40}$. Beyond sperm capacitation, the zinc signature or sperm zinc signaling of spermatozoa bound to the oocyte ZP could be altered by the $\mathrm{Zn}$ spark ${ }^{1,2}$ triggered by the first fertilizing spermatozoon as well be altered by the $300 \%$ increase of zinc content in the $\mathrm{ZP}$ matrix following the $\mathrm{Zn}$ spark $^{41}$. Altogether, such massive release of extracellular $\mathrm{Zn}^{2+}$ and increased $\mathrm{ZP} \mathrm{Zn}$ content could establish a combined zinc shield. Consequently, this zinc shield could derail $\mathrm{Zn}$ signaling in the spermatozoa surrounding the oocyte as an added barrier to polyspermic fertilization. Such mechanism seems plausible in consideration that $\mathrm{Zn}$ has been shown to be chemorepulsive to fertilization-competent human, mouse, and rabbit spermatozoa ${ }^{42}$. Furthermore, acrosin and matrix metalloproteinase MMP2, two of the proteinases implicated in sperm-ZP 

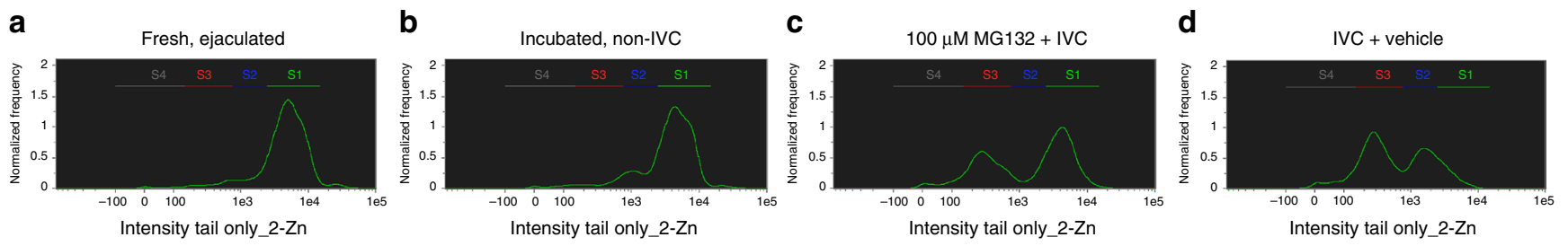

e
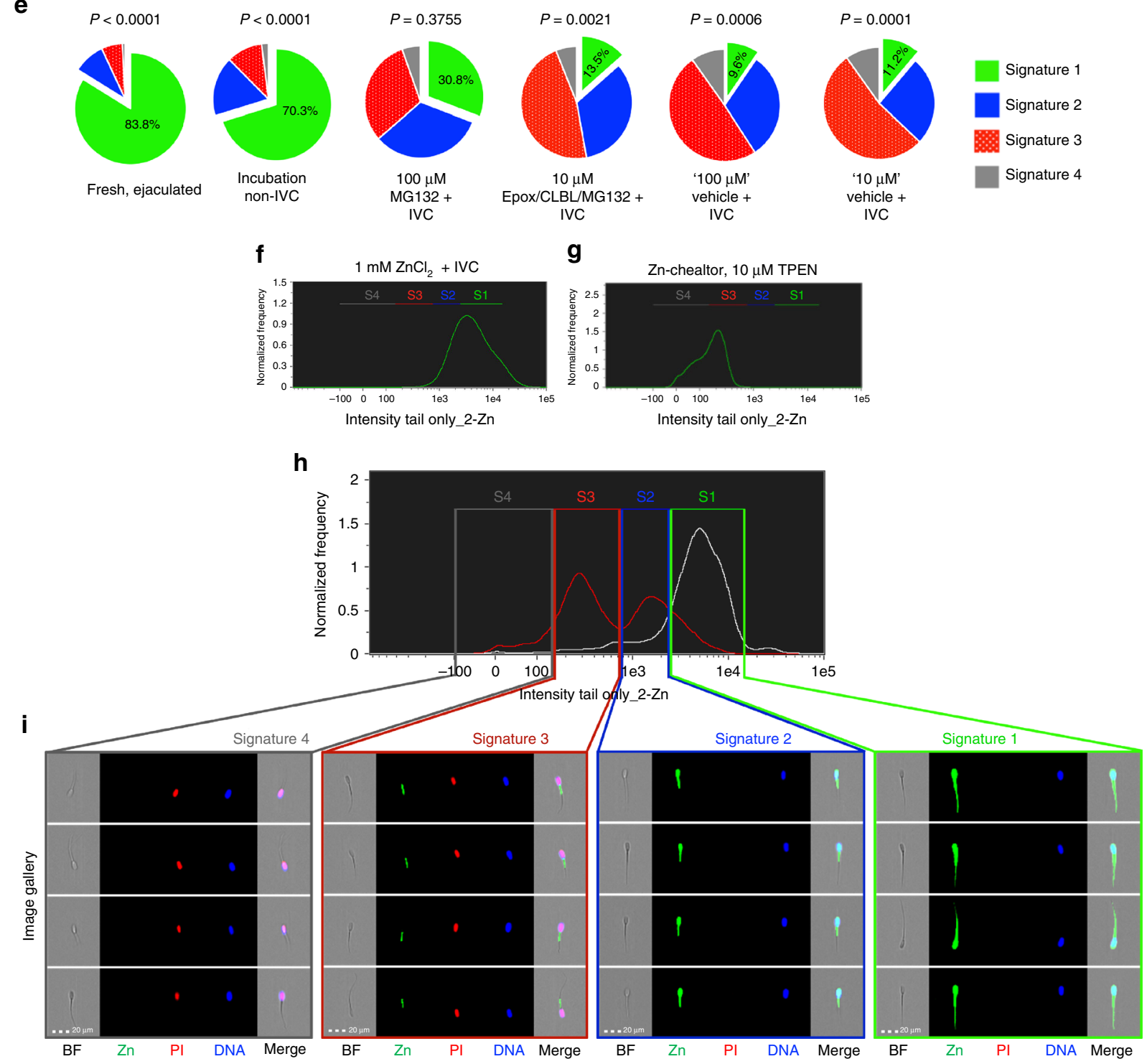

Fig. 4 Modulation of internal and external $\mathrm{Zn}^{2+}$ during IVC. a Fresh, ejaculated spermatozoa have mostly signature 1. b After $4 \mathrm{~h}$ under non-IVC conditions, few spermatozoa underwent spontaneous early-stage capacitation. c Proteasomal inhibitor MG132 (100 $\mu$ M) prevented some of the IVC-induced zinc signature changes compared to d (IVC with MG132-vehicle); see Supplementary Fig. $2 \mathrm{~g}$ for no vehicle IVC control. e Ten-micrometer Zn-chelator TPEN altered the zinc signature; see Supplementary Fig. $2 \mathrm{~h}$ for TPEN vehicle treatment ( $P$-value by treatment across replicates). $\mathbf{f} \mathrm{Addition}_{\mathrm{i}}$ of $1 \mathrm{mM} \mathrm{ZnCl} 2+\mathrm{IVC}$ prevented IVC-induced zinc signature changes. $\mathbf{g}$ Pie chart illustration of select IVC treatments (green: signature 1; blue: signature 2; red: signature 3; gray: signature 4). Treatment $P$-values are shown in Table 2 as determined by the general linear model procedure in SAS 9.4. 10,000 sperm per sample analyzed. $\mathbf{h}$ Histograms of non-capacitated (non-IVC, white) and capacitated (IVC, red) sperm populations from IBFC analysis i Images from IBFC gallery representing individual zinc signatures (scale bar: $20 \mu \mathrm{m}$ ). Each spermatozoon analyzed has the following images acquired: bright field (BF); $Z \mathrm{n}^{2+}$ reporting probe FZ3 (Zn); sperm viability/plasma membrane integrity probe propidium iodide (PI); live DNA stain Hoechst 33342 (DNA); and side scatter (not displayed), with a merger of the four images (Merge)

penetration are present in the inner acrosomal membrane $e^{43}$, (the location believed to be the leading edge of the sperm head during $\mathrm{ZP}$ penetration), have been shown to have their activities reduced and/or inhibited by $\mathrm{Zn}$ (human and bovine sperm acrosin ${ }^{44}$, paddlefish acrosin-like activity ${ }^{45}$, and brain MMP2 activity ${ }^{46}$ ).
Increased $\mathrm{Zn}^{2+}$ concentration in bovine IVF media has previously been shown to inhibit fertilization ${ }^{47}$ adding further support to the effective role of the zinc shield. Based on the present data, sperm zinc signature likely changes as the spermatozoa advance through the female reproductive tract and progress 
Table 2 Effect of proteasomal inhibition on zinc signature

\begin{tabular}{|c|c|c|c|c|c|}
\hline Treatment & Signature 1 & Signature 2 & Signature 3 & Signature 4 & P-value \\
\hline Fresh, ejaculated & $83.8 \pm 1.8 \% \mathrm{Aa}$ & $9.2 \pm 0.9 \%^{b}$ & $6.1 \pm 1.2 \% \mathrm{Ab}$ & $0.9 \pm 0.4 \% A c$ & $P<0.0001$ \\
\hline Incubation, non-IVC & $70.3 \pm 2.5 \% \mathrm{Aa}$ & $17.3 \pm 3.0 \%{ }^{b}$ & $10.4 \pm 2.7 \% \mathrm{Ab}$ & $2.0 \pm 2.7 \% \mathrm{Ac}$ & $P<0.0001$ \\
\hline $100 \mu \mathrm{M}$ MG132 + IVC & $30.8 \pm 13.1 \%{ }^{B}$ & $32.9 \pm 19.5 \%$ & $31.0 \pm 4.9 \%^{B}$ & $5.4 \pm 4.9 \% \mathrm{AB}$ & $P=0.3755$ \\
\hline $10 \mu \mathrm{M}$ Epox, CLBL, MG132 + IVC & $13.5 \pm 4.0 \%^{\mathrm{BC}}$ & $33.7 \pm 8.8 \%$ & $46.8 \pm 3.6 \% \mathrm{C}$ & $6.1 \pm 3.6 \%{ }^{B}$ & $P=0.0021$ \\
\hline "100 $\mu \mathrm{M}$ " Vehicle + IVC & $9.6 \pm 3.3 \% \mathrm{Ca}$ & $31.3 \pm 7.0 \% \mathrm{~b}$ & $49.4 \pm 4.5 \%{ }^{\mathrm{Cc}}$ & $9.8 \pm 4.5 \% \mathrm{Ba}$ & $P=0.0006$ \\
\hline "10 $\mu \mathrm{M} "$ Vehicle + IVC & $11.2 \pm 2.5 \% \mathrm{Ca}$ & $25.8 \pm 3.6 \%{ }^{b}$ & $53.1 \pm 3.8 \% \mathrm{Cc}$ & $9.9 \pm 3.8 \% \mathrm{Ba}$ & $P=0.0001$ \\
\hline$P$-value & $P<0.0001$ & $P=0.4119$ & $P<0.0001$ & $P<0.0003$ & \\
\hline
\end{tabular}

Data are presented as mean \pm s.e.m. (three biological replicates). Values with different uppercase superscripts ( $A, B, C, D$ ) indicate significant difference between the control (fresh, ejaculated spermatozoa and vehicle controls) and treatment groups and lowercase superscripts $(a, b, c)$ indicate significant difference between signatures as determined by Duncan's multiple range test $(P$-value by treatment and signature in table). Both $\mathrm{PI}^{+}$and $\mathrm{PI}^{-}$cells were included in this analysis. Treatment column refers to proteasomal inhibitors MG132, clasto-lactacystin B-Lactone (CLBL), and epoxomicin (Epox.). A total of 10,000 cells were measured for each data point

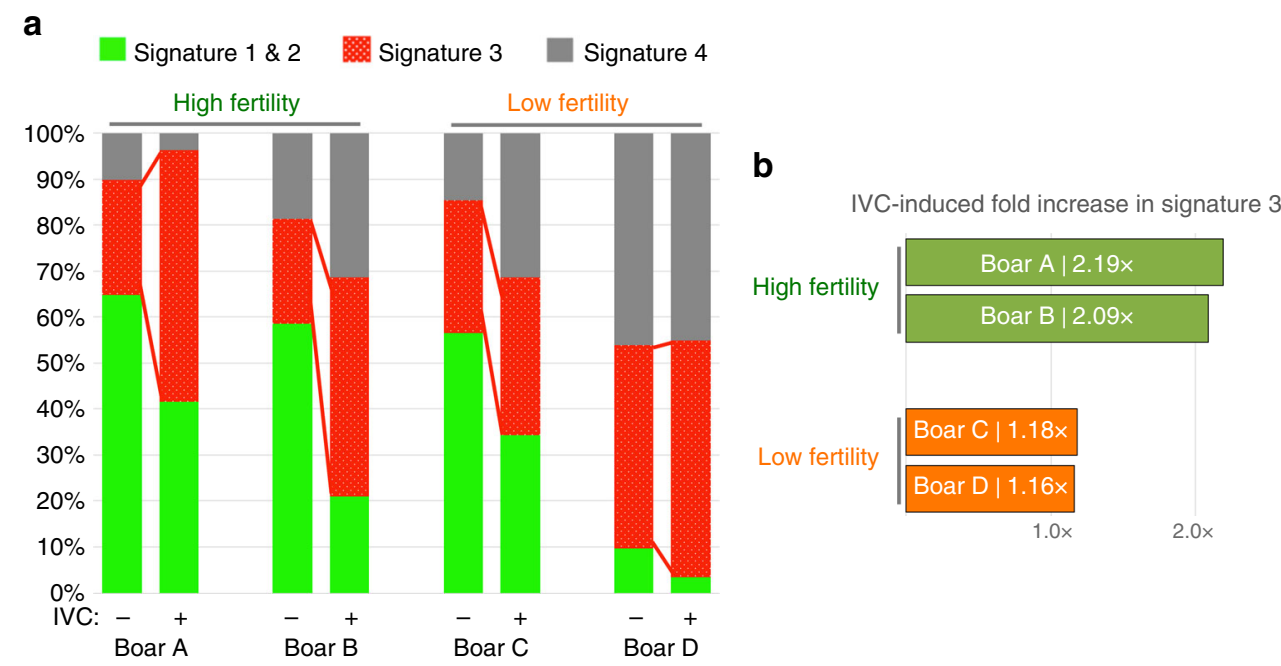

Fig. 5 Zinc signature associated with varied fertility in AI boars. a Zinc signature in four boars of known high or low fertility, before and after IVC. b Highfertility boars had double the amount of spermatozoa with signature 3 after IVC compared to minimal increase in low-fertility boars

through different stages of capacitation; the proposed, reciprocal sperm, and oocyte $\mathrm{Zn}^{2+}$ signaling for the blockage of polyspermy is outlined in Fig. 6b. Lastly, porcine ZP glycoproteins are highly glycosylated with neutral and acidic $\mathrm{N}$ - and $\mathrm{O}$ - linked oligosaccharides. N-glycans released from the ZP3-glycoprotein mixture are composed of neutral and acidic structures in approximate molar ratio of $1: 2^{48}$. While neutral zona $\mathrm{N}$-glycans have been found to be the determining sugar signals in the pig ${ }^{49,50}$, only little is known about the function of acidic moieties of ZP glycoproteins ${ }^{51}$. It is plausible that $\mathrm{Zn}^{2+}$, after $\mathrm{Zn}^{2+}$ efflux associated with gamete fusion, may electrostatically bind to sialic residues of acidic oligosaccharides of ZP, altering ZP polarity and thus act as a fast block of polyspermy on the egg coat level by creating a zinc shield at the time of sperm-oolemma fusion. A similar effect could be achieved by $\mathrm{Zn}^{2+}$ on the sperm surface, but most probably via a different mechanism, such as conformational change of ZP receptors resulting in decreased sperm affinity for the ZP.

Such findings shift the paradigm of anti-polyspermy defense mechanisms and complement the discovery of the oocyte zinc spark. Herein, if capacitation is defined solely as the capacity to fertilize, it would seem reasonable that the oocyte can decapacitate spermatozoa to some degree by manipulating sperm $\mathrm{Zn}$ levels; however, direct relationship with zinc signature should be explored.

In conclusion, the novel biological phenomenon of the sperm zinc signature is an early indicator of sperm capacitation and a candidate biomarker of sperm quality/fertility. The sperm proteasome, at least in the subpopulation of capacitation competent spermatozoa, co-regulates earlier events of sperm capacitation than originally realized, and the Zn-chelator TPEN quenches the sperm zinc signature. The study of sperm zinc signature may further enhance our understanding of fertilization, including but not limited to the oocyte zinc shield formed upon fertilization to prevent polyspermy and sperm subpopulations capable of fertilization.

\section{Methods}

Reagents. All reagents unless otherwise noted were from Sigma. FluoZinn" ${ }^{\mathrm{mw}}-3$, AM (FZ3; zinc probe) from ThermoFisher (F24195) was reconstituted with DMSO to a stock solution of $500 \mu \mathrm{M}$. Lectin PNA (A. hypogea/peanut agglutinin) conjugated to Alexa Fluor 647 (PNA-AF647) was from Invitrogen ${ }^{\mathrm{ntw}}$ (L32460). Fluo-4 NW (calcium probe) from ThermoFisher (F36206) was reconstituted using kit provided assay buffer. Hoechst 33342 (H33342) from Calbiochem (382065) was reconstituted with $\mathrm{H}_{2} \mathrm{O}$ to a stock solution of $18 \mathrm{mM}$. PI from Acros Organics (AC440300010) was reconstituted with $\mathrm{H}_{2} \mathrm{O}$ to a stock solution of $1 \mathrm{mg} \mathrm{mL} \mathrm{mL}^{-1}$. Proteasomal inhibitors were from Enzo Life Sciences: MG132 (BML-PI102) was reconstituted with DMSO to a stock solution of $20 \mathrm{mM}$; epoxomicin (Epox, BMLPI127) was reconstituted to a stock solution of $20 \mathrm{mM}$ (using MG132 stock); and clasto-lactacystin $\beta$-Lactone (CLBL, BML-PI108) was reconstituted with DMSO to a stock solution of $5 \mathrm{mM}$. Zn-chelator TPEN from Tocris (16858-02-9) was resuspended with 1:100 EtOH: $\mathrm{H}_{2} \mathrm{O}$ to a stock solution of $1 \mathrm{mM}$. Bovine serum albumin was from Sigma (A4503). Anti-phosphotyrosine antibody, clone $4 \mathrm{G} 10^{\circ}$ was from EMD Millipore (05-321).

Semen collection and processing. Boar semen collection was performed under the guidance of approved Animal Care and Use (ACUC) protocols of the University of Missouri-Columbia. Boar semen for the fertility trial was collected, extended, and shipped by overnight parcel from a private boar stud following their 
a

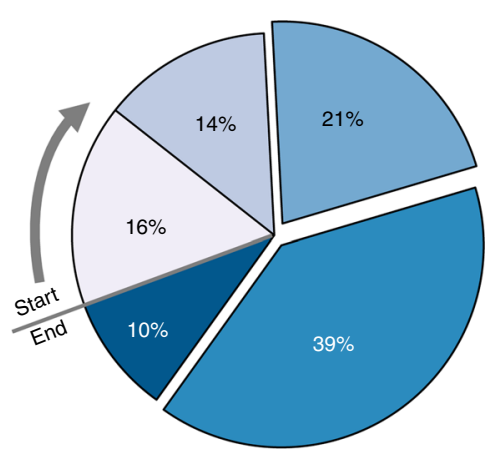

Key

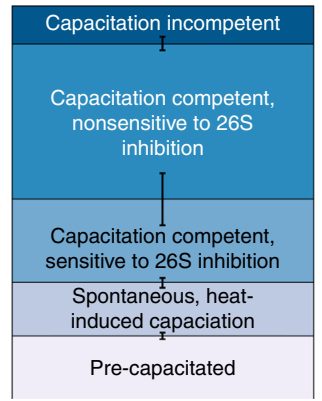

b

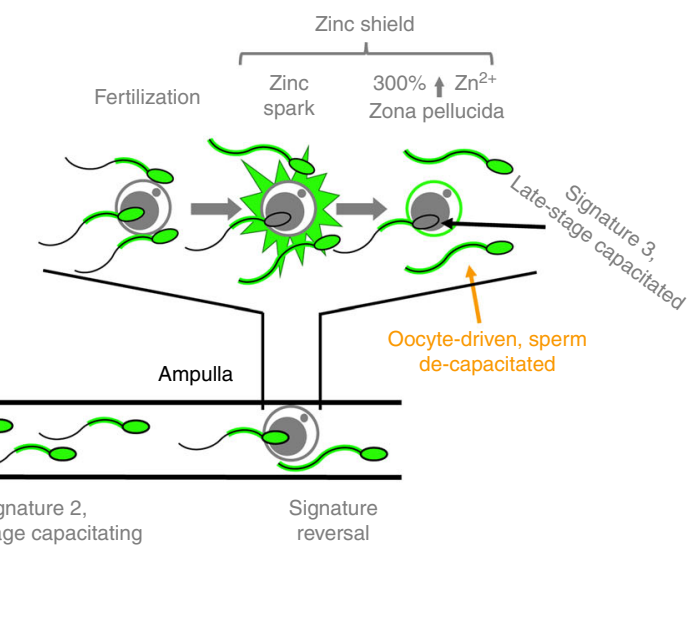

Fig. 6 Proposed zinc signature population interpretation. a Interpretation of zinc signature meaning and population segregation: 16\% of fresh, ejaculated spermatozoa had undergone early-stage capacitation upon semen collection (lightest blue working to darkest); $14 \%$ of spermatozoa spontaneously undergo early-stage capacitation during incubation without IVC inducers; $60 \%$ of spermatozoa remained capacitation competent with IVC inducers, with $21 \%$ sensitive to proteasomal inhibition; remaining $10 \%$ of sperm were capacitation incompetent under IVC conditions (darkest blue) (s.e. bars included). b Proposed zinc signature changes throughout female reproductive tract and oocyte zinc spark interference with sperm zinc signature as a combined polyspermy defense mechanism, the zinc shield

established standard operating procedures and was not blinded. Boar collection was performed using standard two gloved hand technique ${ }^{9}$. Only ejaculates with $>80 \%$ motility were used and no randomization was necessary as only one boar was studied at a time. The sperm-rich fraction of the boar ejaculate was used, except all IVC proteasomal inhibition studies used the pre-sperm-rich fraction, which had increased viability and sensitivity to $26 \mathrm{~S}$ inhibition. Semen was immediately extended, within $2{ }^{\circ} \mathrm{C}$, five times in BTS semen extender. Sperm concentration was then determined using a hemocytometer. All washes were performed with a swing hinge rotor centrifuge at $110 \times g$ for $5 \mathrm{~min}$. The number of washes and $\mathrm{g}$-force used were minimized as these were found to compromise results. Frozen-thawed bull spermatozoa were processed similarly as boar spermatozoa after being thawed for $45 \mathrm{~s}$ in a $35^{\circ} \mathrm{C}$ water bath. For human spermatozoa, sperm donors signed informed consent and the samples were coded as to make the donors unidentifiable to researchers. All human sperm samples were handled and processed strictly as stipulated by an approved Internal Review Board (MU IRB) protocol. Donors were recruited by placing an advertisement for new fathers in the university mass e-mail newsletter. All semen were collected onsite at the Missouri Center for Reproductive Medicine and Fertility clinic. Samples were then transported to the laboratory for analysis.

In vitro capacitation. Fresh boar spermatozoa were capacitated using a protocol that rendered them capable of recognizing and binding to $\mathrm{ZP}$, as well as undergoing acrosomal exocytosis and penetrating the oocyte $\mathrm{ZP}^{11}$. IVC-induced protein tyrosine phosphorylation changes are shown in Supplementary Fig. 5; acrosomal status and plasma membrane changes are shown in Fig. 2. Briefly, spermatozoa were washed of seminal plasma once with noncapacitating media (NCM), a modified TLHEPES medium, free of calcium dichloride $\left(\mathrm{CaCl}_{2}\right)$ and addition of $11 \mathrm{mM} \mathrm{D}-$ glucose, with $\mathrm{pH}$ adjusted to 7.2 . Spermatozoa were then resuspended in $0.5 \mathrm{~mL}$ IVC media, TL-HEPES-PVA supplemented with $5 \mathrm{mM}$ sodium pyruvate, $11 \mathrm{mM} \mathrm{D-}$ glucose, $2 \mathrm{mM} \mathrm{CaCl}, 2 \mathrm{mM}$ sodium bicarbonate, and $2 \%(\mathrm{~m} / \mathrm{v})$ bovine serum albumin, and incubated in a $37^{\circ} \mathrm{C}$ water bath for $4 \mathrm{~h}$, with eppendorf tube rotation performed every $60 \mathrm{~min}$. Control incubations under non-IVC conditions used NCM. Proteasome inhibitors (100 $\mu \mathrm{M}$ MG-132 and $10 \mu \mathrm{M}$ Epox/CLBL/MG-132) were mixed with IVC media prior to sperm pellet resuspension. A $100 \mu \mathrm{M}$ MG-132 and " $100 \mu \mathrm{M}$ " vehicle contained $0.5 \%$ (v/v) DMSO. A $10 \mu \mathrm{M}$ Epox/CLBL/MG-132 and " $10 \mu \mathrm{M}$ " vehicle contained $0.3 \%(\mathrm{v} / \mathrm{v})$ DMSO. PVA helped to reduce sperm aggregation and spermatozoa were pipetted repeatedly to dissociate sperm aggregates in a satisfactory manner prior to IBFC data acquisition. To confirm normal capacitation in our experimental IVC media, compared to $15 \mathrm{mM}$ sodium bicarbonate IVC media, we refer readers to Supplementary Movie 1 (hyperactivation status) and Supplementary Fig. 5 (tyrosine phosphorylation status). Both media support hyperactivated motility. Unlike murine or rodent sperm tyrosine phosphorylation, porcine tyrosine phosphorylation is much more modest, with less prominent changes during the course of capacitation. Therefore, new bands after capacitation appear only at the molar weights of $32 \mathrm{kDa}$ (acrosin-binding protein), and $21 \mathrm{kDa}$ protein (phospholipid hydroperoxide glutathione peroxidase). Our results are in accordance with previous studies ${ }^{52-54}$. Final acrosome and plasma membrane modification status is similar at the end of IVC regardless of the two IVC treatment conditions; however, the rate of change and cell death is faster in $15 \mathrm{mM}$ sodium bicarbonate-containing medium than experimental IVC medium

(Fig. 2a-d). Altogether, this supports the use of experimental IVC medium over 15 $\mathrm{mM}$ sodium bicarbonate-containing medium to display the prolonged lifespan of spermatozoa as seen in in vivo capacitation.

TPEN Zn chelation. $\mathrm{Zn}^{2+}$ chelation was performed using TPEN (membrane permeable). Ten micromolar TPEN was incubated with 40 million sperm per $\mathrm{mL}$ for $1 \mathrm{~h}$. Stock TPEN: $1 \mathrm{mM}$ in 1:100 EtOH: $\mathrm{H}_{2} \mathrm{O}$.

Multiplex fluorescence probing. Upon $4 \mathrm{~h}$ of IVC, a sample size of $100 \mu \mathrm{L}$ (4 million spermatozoa) were incubated 30 min with 1:200 H33342, 1:200 PI, and 1:100 FZ3 for epifluorescence microscopy. Lower probe concentrations were necessary for IBFC due to camera detection differences, thus 1:1000, 1:1000, and 
1:500 were used, respectively, with inclusion of 1:1000 PNA-AF647. For Fluo-4 calcium probe, we followed manufacturer protocol using identical cell concentrations. Spermatozoa were then washed of probes once and resuspended in corresponding IVC treatment media to allow complete de-esterfication of intracellular AM esters, as suggested by ThermoFisher's FZ3 protocol, followed by an additional wash and resuspended in $100 \mu \mathrm{L}$ PBS for IBFC analysis (or added to a slide for epifluorescence microscopy imaging).

Epifluorescence microscopy imaging. Live spermatozoa were imaged using a Nikon Eclipse 800 microscope (Nikon Instruments Inc.) with Cool Snap camera (Roper Scientific, Tuscon, AZ, USA) and MetaMorph software (Universal Imaging Corp., Downington, PA, USA). Images were adjusted for contrast and brightness in Adobe Photoshop CS5 (Adobe Systems, Mountain View, CA) to match the fluorescence intensities viewed through the microscope eyepieces.

Image-based flow cytometric data acquisition. IBFC data acquisition was performed following previous methodology ${ }^{55}$. Specifically, using a FlowSight flow cytometer (FS) fitted with a $\times 20$ microscope objective (numerical aperture of 0.9 ) with an imaging rate up to 2000 events per s. The sheath fluid was PBS (without $\mathrm{Ca}^{2+}$ or $\left.\mathrm{Mg}^{2+}\right)$. The flow-core diameter and speed was $10 \mu \mathrm{m}$ and $66 \mathrm{~mm}$ per s, respectively. Raw image data were acquired using INSPIRE software. To produce the highest resolution, the camera setting was at $1.0 \mu \mathrm{m}$ per pixel of the chargedcoupled device. In INSPIRE ${ }^{\circledR}$ FS data acquisition software, two bright-field channels were collected (channels 1 and 9), one FZ3 image (channel 2), one PI image (channel 5), one side scatter (SSC; channel 6), one H33342 (channel 7), and one PNA-AF647 image (channel 11), with a minimum of 10,000 spermatozoa collected. The following lasers and power settings were used: $405 \mathrm{~nm}$ (to excite H33342): 10 $\mathrm{mW}$; $488 \mathrm{~nm}$ (to excite FZ3): $60 \mathrm{~mW}$; $561 \mathrm{~nm}$ (to excite PI): $40 \mathrm{~mW}, 642 \mathrm{~nm}$ (to excite PNA-AF647): $25 \mathrm{~mW}$; and $785 \mathrm{nM}$ SSC laser: $10 \mathrm{~mW}$.

IBFC data analysis. Data were analyzed using IDEAS ${ }^{\bullet}$ analysis software from AMNIS EMD Millipore. Gating approach used standard focus and single cell gating calculations created by IDEAS software (Supplementary Fig. 1a, b). To further clean up data for analysis, Feature Finder function was used to discover image-based calculations to discard spermatozoa laterally aligned with the camera, as opposed to anteriorly/posteriorly aligned (Supplementary Fig. 1c). Traditional flow cytometric analysis methods does not allow to distinguish signature 1 and 2 based on whole-cell FZ3 intensity, therefore creating a mask that only analyzes the sperm tail proved to be key in distinguishing these two populations. Such mask was created by taking a morphology mask of the bright field (Supplementary Fig. 1d), subtracting a 4-pixel dilation of H33342 (Supplementary Fig. 1e), resulting in a mask to analyze fluorescence in the tail region only (Supplementary Fig. If, g). Mask dilation of $\mathrm{H} 33342$ fluorescence was necessary because H33342 labeling of the sperm nucleus did not cover the entirety of the sperm head, where FZ3 signal was high. This combined gating and masking strategy provided robust clean data, ready for signature analysis by plotting FZ3 intensity of this masked region, which is impossible with traditional flow cytometry. Gating boundaries for signatures 1, 2, and 3 were determined by the population segregations of fresh/ejaculate and IVC + vehicle treatments with signature status confirmed in the image gallery. Gating boundaries between signatures 3 and 4 were less evident in histograms and placed where spermatozoa lost FZ3 signal as determined using the image gallery.

Sequential sperm extraction treatment. Approximately 200 million washed spermatozoa were used per single treatment, which was conducted by adding 100 $\mu \mathrm{L}$ of a relevant reagent; proteinase and phosphatase inhibitors included. In the first step, PBS was added to the sperm pellet, allowed to incubate on ice for $30 \mathrm{~min}$ and spun. In second step, the pellet was reused and $0.75 \mathrm{M} \mathrm{KCl}$ in PBS was added and incubated on ice for $30 \mathrm{~min}$ and spun down. The pellet was washed once with PBS to remove residual salt and reused in the third step for treatment with $30 \mathrm{mM}$ $n$-octyl- $\beta$-D-glucopyranoside (OBG) in PBS. The sperm after each treatment step were analyzed for their zinc signature.

Western blotting. Sperm pellets (15 million spermatozoa per pellet) were mixed with reducing SDS-PAGE loading buffer, boiled for $5 \mathrm{~min}$ and briefly spun at $5000 \times g$. The SDS-PAGE was carried out on a $4-20 \%$ gradient gels (PAGEr Precast gels; Lonza Rockland, Rockland, ME, USA) as previously described ${ }^{13}$.The molecular masses of the separated proteins were estimated by using prestained Prosieve protein colored markers (Lonza Rockland) run in parallel. After SDS-PAGE, proteins were electro-transferred onto a PVDF Immobilon Transfer Membrane (Millipore, Bedford, MA, USA) using an Owl wet transfer system (Fisher Scientific) at a constant $50 \mathrm{~V}$ for $4 \mathrm{~h}$ for immunodetection ${ }^{13}$

Statistics. All results are presented as mean \pm s.e. SAS 9.4 GLM procedure and Duncan's multiple range test was used to analyze the replicates. Bartlett and Leven tests found the sample set to be homogenous.
Animal models. Domestic (Sus scrofa) boars 2 years of age were used for all experiments performed. Domestic (Bos taurus) bulls 3 years of age were used to confirm zinc signature presence.

Data availability. There are no database deposited data in this article. All relevant data are available from the authors.

Received: 9 June 2017 Accepted: 2 May 2018

Published online: 25 May 2018

\section{References}

1. Que, E. L. et al. Quantitative mapping of zinc fluxes in the mammalian egg reveals the origin of fertilization-induced zinc sparks. Nat. Chem. 7, 130-139 (2015).

2. Zhang, N., Duncan, F. E., Que, E. L., O’Halloran, T. V. \& Woodruff, T. K. The fertilization-induced zinc spark is a novel biomarker of mouse embryo quality and early development. Sci. Rep. 6, 22772 (2016).

3. Lishko, P. V., Botchkina, I. L., Fedorenko, A. \& Kirichok, Y. Acid extrusion from human spermatozoa is mediated by flagellar voltage-gated proton channel. Cell 140, 327-337 (2010).

4. Tardif, S., Dube, C. \& Bailey, J. L. Porcine sperm capacitation and tyrosine kinase activity are dependent on bicarbonate and calcium but protein tyrosine phosphorylation is only associated with calcium. Biol. Reprod. 68, 207-213 (2003)

5. Boerke, A., Tsai, P. S., Garcia-Gil, N., Brewis, I. A. \& Gadella, B. M. Capacitation-dependent reorganization of microdomains in the apical sperm head plasma membrane: functional relationship with zona binding and the zona-induced acrosome reaction. Theriogenology 70, 1188-1196 (2008).

6. Sutovsky, P. Sperm proteasome and fertilization. Reproduction 142, 1-14 (2011).

7. Kerns, K., Morales, P. \& Sutovsky, P. Regulation of sperm capacitation by the $26 \mathrm{~S}$ proteasome: an emerging new paradigm in spermatology. Biol. Reprod. 94, 117 (2016).

8. Hillman, P., Ickowicz, D., Vizel, R. \& Breitbart, H. Dissociation between AKAP3 and PKARII promotes AKAP3 degradation in sperm capacitation. PLoS ONE 8, e68873 (2013).

9. Yi, Y. J. et al. Ubiquitin-activating enzyme (UBA1) is required for sperm capacitation, acrosomal exocytosis and sperm-egg coat penetration during porcine fertilization. Int. J. Androl. 35, 196-210 (2012).

10. Jaiswal, B. S. \& Eisenbach, M. in Fertilization (ed Daniel M. Hardy) Ch. 3, 57-117 (Academic Press, San Diego, 2002).

11. Zimmerman, S. W. et al. Sperm proteasomes degrade sperm receptor on the egg zona pellucida during mammalian fertilization. PLOS ONE 6, e17256 (2011).

12. Yi, Y. J. et al. Sperm-surface ATP in boar spermatozoa is required for fertilization: relevance to sperm proteasomal function. Syst. Biol. Reprod. Med. 55, 85-96 (2009).

13. Miles, E. L. et al. Transgenic pig carrying green fluorescent proteasomes. Proc Natl Acad. Sci. USA 110, 6334-6339 (2013).

14. Stoltenberg, M., Sørensen, M. \& Danscher, G. Histochemical demonstration of zinc ions in ejaculated human semen. Int. J. Androl. 20, 229-236 (1997).

15. Zimmerman, S. W. et al. Identification and characterization of RING-finger ubiquitin ligase UBR7 in mammalian spermatozoa. Cell Tissue Res. 356, 261-278 (2014).

16. Bianchi, F., Rousseaux-Prevost, R., Sautiere, P. \& Rousseaux, J. P2 protamines from human sperm are zinc -finger proteins with one CYS2/HIS2 motif. Biochem. Biophys. Res. Commun. 182, 540-547 (1992).

17. Nanassy, L., Liu, L., Griffin, J. \& Carrell, D. T. The clinical utility of the protamine 1/protamine 2 ratio in sperm. Protein Pept. Lett. 18, 772-777 (2011).

18. Ambroggio, X. I., Rees, D. C. \& Deshaies, R. J. JAMM: a metalloprotease-like zinc site in the proteasome and signalosome. PLoS Biol. 2, e2 (2003).

19. Nagase, H. \& Woessner, J. F. Jr. Matrix metalloproteinases. J. Biol. Chem. 274, 21491-21494 (1999).

20. Shimokawa Ki, K. et al. Matrix metalloproteinase (MMP)-2 and MMP-9 activities in human seminal plasma. Mol. Hum. Reprod. 8, 32-36 (2002).

21. Beek, J., Nauwynck, H., Maes, D. \& Van Soom, A. Inhibitors of zincdependent metalloproteases hinder sperm passage through the cumulus oophorus during porcine fertilization in vitro. Reproduction 144, 687-697 (2012).

22. Bjorndahl, L. \& Kvist, U. Human sperm chromatin stabilization: a proposed model including zinc bridges. Mol. Hum. Reprod. 16, 23-29 (2010).

23. Kvist, U. Importance of spermatozoal zinc as temporary inhibitor of sperm nuclear chromatin decondensation ability in man. Acta Physiol. Scand. 109, 79-84 (1980) 
24. Holt, W. V. \& North, R. D. Thermotropic phase transitions in the plasma membrane of ram spermatozoa. J. Reprod. Fertil. 78, 447-457 (1986).

25. Qi, H. et al. All four CatSper ion channel proteins are required for male fertility and sperm cell hyperactivated motility. Proc. Natl Acad. Sci. USA 104, 1219-1223 (2007).

26. Buffone, M. G., Calamera, J. C., Verstraeten, S. V. \& Doncel, G. F. Capacitation-associated protein tyrosine phosphorylation and membrane fluidity changes are impaired in the spermatozoa of asthenozoospermic patients. Reproduction 129, 697-705 (2005).

27. Suarez, S. S. Regulation of sperm storage and movement in the mammalian oviduct. Int. J. Dev. Biol. 52, 455-462 (2008)

28. Leemans, B. et al. An alkaline follicular fluid fraction induces capacitation and limited release of oviduct epithelium-bound stallion sperm. Reproduction 150, 193-208 (2015).

29. Giojalas, L. C., Rovasio, R. A., Fabro, G., Gakamsky, A. \& Eisenbach, M. Timing of sperm capacitation appears to be programmed according to egg availability in the female genital tract. Fertil. Steril. 82, 247-249 (2004).

30. Andreychenko, S. V. et al. Post-chornobyl remote radiation effects on human sperm and seminal plasma characteristics. Exp. Oncol. 38, 245-251 (2016).

31. Nenkova, G., Petrov, L. \& Alexandrova, A. Role of trace elements for oxidative status and quality of human sperm. Balkan Med. J. 34, 343-348 (2017).

32. Colagar, A. H., Marzony, E. T. \& Chaichi, M. J. Zinc levels in seminal plasma are associated with sperm quality in fertile and infertile men. Nutr. Res. 29, 82-88 (2009).

33. Zhao, J. et al. Zinc levels in seminal plasma and their correlation with male infertility: a systematic review and meta-analysis. Sci. Rep. 6, 22386 (2016).

34. Harrison, R. A., Dott, H. M. \& Foster, G. C. Effect of ionic strength, serum albumin and other macromolecules on the maintenance of motility and the surface of mammalian spermatozoa in a simple medium. J. Reprod. Fertil. 52, 65-73 (1978)

35. Beyersmann, D. \& Haase, H. Functions of zinc in signaling, proliferation and differentiation of mammalian cells. Biometals 14, 331-341 (2001).

36. Kim, I. et al. Pyrrolidine dithiocarbamate and zinc inhibit proteasomedependent proteolysis. Exp. Cell Res. 298, 229-238 (2004).

37. Massanyi, P. et al. Concentration of copper, iron, zinc, cadmium, lead, and nickel in boar semen and relation to the spermatozoa quality. J. Environ. Sci. Health A Tox Hazard. Subst. Environ. Eng. 38, 2643-2651 (2003).

38. Mogielnicka-Brzozowska, M., Wysocki, P., Strzezek, J. \& Kordan, W. Zincbinding proteins from boar seminal plasma -- isolation, biochemical characteristics and influence on spermatozoa stored at $4{ }^{\circ} \mathrm{C}$. Acta Biochim. Pol. 58, 171-177 (2011)

39. Henkel, R., Bittner, J., Weber, R., Hüther, F. \& Miska, W. Relevance of zinc in human sperm flagella and its relation to motility. Fertil. Steril. 71, 1138-1143 (1999).

40. Sørensen, M. B., Stoltenberg, M., Danscher, G. \& Ernst, E. Chelation of intracellular zinc ions affects human sperm cell motility. Mol. Hum. Reprod. 5, 338-341 (1999).

41. Que, E. L. et al. Zinc sparks induce physiochemical changes in the egg zona pellucida that prevent polyspermy. Integr. Biol. 9, 135-144 (2017).

42. Guidobaldi, H. A. et al. Sperm chemorepulsion, a supplementary mechanism to regulate fertilization. Hum. Reprod. 32, 1560-1573 (2017).

43. Ferrer, M. et al. MMP2 and acrosin are major proteinases associated with the inner acrosomal membrane and may cooperate in sperm penetration of the zona pellucida during fertilization. Cell Tissue Res. 349, 881-895 (2012).

44. Steven, F. S., Griffin, M. M. \& Chantler, E. N. Inhibition of human and bovine sperm acrosin by divalent metal ions. Possible role of zinc as a regulator of acrosin activity. Int. J. Androl. 5, 401-412 (1982).

45. Ciereszko, A., Dabrowski, K., Mims, S. D. \& Glogowski, J. Characteristics of sperm acrosin-like activity of paddlefish (Polyodon spathula Walbaum). Comp. Biochem. Physiol. B Biochem. Mol. Biol. 125, 197-203 (2000).

46. Backstrom, J. R., Miller, C. A. \& Tokes, Z. A. Characterization of neutral proteinases from Alzheimer-affected and control brain specimens: identification of calcium-dependent metalloproteinases from the hippocampus. J. Neurochem. 58, 983-992 (1992).

47. Stephenson, J. L. \& Brackett, B. G. Influences of zinc on fertilisation and development of bovine oocytes in vitro. Zygote 7, 195-201 (1999).

48. Noguchi, S. \& Nakano, M. Structure of the acidic N-linked carbohydrate chains of the $55-\mathrm{kDa}$ glycoprotein family (PZP3) from porcine zona pellucida. Eur. J. Biochem. 209, 883-894 (1992).
49. Yurewicz, E. C., Pack, B. A. \& Sacco, A. G. Isolation, composition, and biological activity of sugar chains of porcine oocyte zona pellucida $55 \mathrm{~K}$ glycoproteins. Mol. Reprod. Dev. 30, 126-134 (1991).

50. Noguchi, S., Hatanaka, Y., Tobita, T. \& Nakano, M. Structural analysis of the $\mathrm{N}$-linked carbohydrate chains of the $55-\mathrm{kDa}$ glycoprotein family (PZP3) from porcine zona pellucida. Eur. J. Biochem. 204, 1089-1100 (1992).

51. Amari, S. et al. Essential role of the nonreducing terminal alpha-mannosyl residues of the $\mathrm{N}$-linked carbohydrate chain of bovine zona pellucida glycoproteins in sperm-egg binding. Mol. Reprod. Dev. 59, 221-226 (2001).

52. Flesch, F. M., Colenbrander, B., van Golde, L. M. G. \& Gadella, B. M. Capacitation induces tyrosine phosphorylation of proteins in the boar sperm plasma membrane. Biochem. Biophys. Res. Commun. 262, 787-792 (1999).

53. Tardif, S., Dube, C., Chevalier, S. \& Bailey, J. L. Capacitation is associated with tyrosine phosphorylation and tyrosine kinase-like activity of pig sperm proteins. Biol. Reprod. 65, 784-792 (2001).

54. Dube, C., Tardif, S., LeClerc, P. \& Bailey, J. L. The importance of calcium in the appearance of $\mathrm{p} 32$, a boar sperm tyrosine phosphoprotein, during in vitro capacitation. J. Androl. 24, 727-733 (2003).

55. Kennedy, C. E. et al. Protein expression pattern of PAWP in bull spermatozoa is associated with sperm quality and fertility following artificial insemination. Mol. Reprod. Dev. 81, 436-449 (2014).

\section{Acknowledgements}

Supported by the National Institute of Food and Agriculture (NIFA), U.S. Department of Agriculture (USDA) grant number 2015-67015-23231 (P.S.), USDA NIFA Graduate Fellowship award number 2017-67011-26023 (K.K.), grant number 5 R01 HD084353-02 from NIH National Institute of Child and Human Development (P.S.), European Regional Development Fund (ERDF) BIOCEV grant CZ.1.05/1.1.00/02.0109 (M.Z.), the Czech Academy of Sciences (RVO:866525036) (M.Z.) and seed funding from the Food for the 21st Century Program of the University of Missouri (P.S.). We thank staff of the National Swine Research Resource Center, University of Missouri, as well as Dr. Randall Prather and his associates for assisting in boar semen collection and processing. We also thank Mr. Dalen Zuidema for providing MII porcine oocytes, Ms. Lauren Mayo for assistance with statistical analysis, and Ms. Katherine Craighead for clerical support.

\section{Author contributions}

K.K. and P.S. designed the research; K.K., M.Z., E.Z.D., M.S., and P.S. performed the research; K.K., M.Z., and P.S. analyzed the data; K.K., M.Z., E.Z.D., and P.S. wrote the paper.

\section{Additional information}

Supplementary Information accompanies this paper at https://doi.org/10.1038/s41467018-04523-y.

\section{Competing interests: The authors declare no competing interests.}

Reprints and permission information is available online at http://npg.nature.com/ reprintsandpermissions/

Publisher's note: Springer Nature remains neutral with regard to jurisdictional claims in published maps and institutional affiliations.

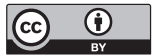

Open Access This article is licensed under a Creative Commons Attribution 4.0 International License, which permits use, sharing, adaptation, distribution and reproduction in any medium or format, as long as you give appropriate credit to the original author(s) and the source, provide a link to the Creative Commons license, and indicate if changes were made. The images or other third party material in this article are included in the article's Creative Commons license, unless indicated otherwise in a credit line to the material. If material is not included in the article's Creative Commons license and your intended use is not permitted by statutory regulation or exceeds the permitted use, you will need to obtain permission directly from the copyright holder. To view a copy of this license, visit http://creativecommons.org/ licenses/by/4.0/.

(c) The Author(s) 2018 